\title{
BATTERI PATOGENI E LORO PROFILI DI RESISTENZA IN PAZIENTI RICOVERATI AFFETTI DA ULCERE CUTANEE CRONICHE A VARIA EZIOLOGIA DEGLI ARTI INFERIORI: STUDIO MICROBIOLOGICO ED EPIDEMIOLOGICO NELLA CLINICA DERMATOLOGICA DI PAVIA.
}

Piccolo C.; Vassallo C.; Gabba P.; Albertuzzi S.; Cananzi R.; Borroni G.

\section{Clinica Dermatologica dell'Università di Pavia,}

Policlinico San Matteo IRCCS, Pavia

Le ulcere cutanee a varia eziologia, in modo particolare degli arti inferiori, e le ulcere da decubito rappresentano un'affezione molto comune, invalidante, caratterizzata da cronicità, guarigione lenta o nulla e causa di limitazione e sofferenza per il paziente.

Si calcola che circa 1'1\% della popolazione italiana ne sia affetta. Si tratta di una patologia socialmente impegnativa e gravosa dal punto di vista economico non solo per le famiglie, ma anche per il Sistema Sanitario Nazionale.

Molteplici sono le presentazioni cliniche ed i meccanismi patogenetici, ma in ogni caso, le ulcere croniche degli arti inferiori vanno incontro ad una complicanza costante: la sovrainfezione batterica.

Diretta conseguenza della presenza di batteri nelle ulcere cutanee croniche è l'ostacolo alla formazione del tessuto di granulazione presupposto indispensabile per la guarigione medica e/o chirurgica.

Scopo del presente lavoro è analizzare la prevalenza degli agenti patogeni riscontrati nelle ulcere cutanee croniche degli arti inferiori a diversa etiopatogenesi in 31 pazienti ricoverati nel Reparto della Clinica Dermatologica di Pavia nel periodo di tempo compreso fra il 2000 e il 2002. Ci si propone inoltre di descrivere l'evoluzione dell'antibioticoresistenza emergente dall'analisi dei 66 antibiogrammi effettuati nel medesimo lasso di tempo nei pazienti ricoverati per tale patologia.

L'agente patogeno Pseudomonas aeruginosa è stato riscontrato in 16 pazienti e in 34 dei 66 tamponi cutanei presi in esame, delineando una prevalenza in entrambi i gruppi del $51,5 \%$.

In tutti gli antibiogrammi considerati Pseudomonas aeruginosa si è rivelato resistente a 5 o più degli antibiotici testati. Il secondo battere patogeno riscontrato per frequenza è stato Staphilococcus aureus, presente in 15 dei 31 pazienti e in 17 tamponi cutanei; la prevalenza nei due gruppi considerati è rispettivamente del $48,4 \%$ e del $25,8 \%$.

In 7 degli antibiogrammi considerati Staphyloccus aureus si è rivelato resistente a 5 o più degli antibiotici testati (prevalenza di multiresistenza: 41,2\%).

In minima percentuale, infine, si è riscontrata la presenza di batteri Gram - non fermentanti: 2 Klebsiella oxytoca, 1 Proteus vulgaris, 3 Proteus mirabilis, 2 Serratia marcescens, 2 Escherichia coli, 3 Morganella morganii e di 1 ceppo Gram - fermentante di Kluyvera ascorbata.

Tra i Gram +, oltre Staphilococcus aureus, 1 ceppo di Streptococcus agalactiae. 